\title{
Outcome of a 12-week programme for management of the spinal cord injured with participation of patient's relations at Hilltop Orthopaedic Hospital, Enugu, Nigeria
}

\author{
OE Nwankwo*,1 and AU Katchy ${ }^{2}$ \\ ${ }^{1}$ University of Nigeria Teaching Hospital, Enugu/Hilltop Orthopaedic Hospital, Trans-Ekulu, Enugu, Enugu State, \\ Nigeria; ${ }^{2}$ National Orthopaedic Hospital, Enugu/Hilltop Orthopaedic Hospital, Trans-Ekulu, Enugu, Enugu State, \\ Nigeria
}

\begin{abstract}
Study design: Description of 12-week programme of treatment. Objectives: In Enugu, Nigeria, not all spinal injured (SCI) can be treated in teaching and orthopaedic hospitals. The 12-week programme was developed with the aim of getting such patients back into society as useful and independent as possible, using the patients relations to complement the deficiency in personnel.

Setting: Enugu, Nigeria.

Methods: Staged weekly programmes of assessment and treatment were mapped out to end at the twelfth week. Spinal injured patients of less than 2 weeks duration were entered into these programmes as they presented. Outcome was assessed at the end of the twelfth week. The study lasted for 6 years (January 1996 to December 2000).

Results: Seventy-four patients were admitted into the study. Nine patients had incomplete cord injury of Frankel $\mathrm{C}$ while 65 had clinically complete injury of Frankel A on admission. Eight of the nine incomplete injuries improved to Frankel D and walked home within the 12week programme. In the clinically complete group, eight died. Out of the 57 remaining, six recovered to Frankel D and walked home, while the remaining 51 who did not recover, 49 were conversant with wheelchair use within the 12-week programme.

Conclusion: It is concluded that adoption of this programme would allow the spinal cord injured to get expert treatment within a reasonable time in less ideal hospital settings where man-power and skilled members of the spinal cord injury treatment team are lacking.

Spinal Cord (2003) 41, 129-133. doi:10.1038/sj.sc.3101410
\end{abstract}

Keywords: spinal cord injured; management; outcome

\section{Introduction}

Spinal cord injury is a mortal condition and has been recognised as such since antiquity. ${ }^{1}$ The chilling advice of an unknown Egyptian physician regarding traumatic tetraplegia in Edwin Smith's papyrus in 2500 BC is that it is 'an ailment not to be treated'.

The view prevailed until the early years of last century. Fortunately, the vision of early pioneers like Guttmann ${ }^{1}$ in the United Kingdom together with Munro $^{1}$ and Bors ${ }^{1}$ in the USA has greatly improved the outlook for those with spinal cord injury.,3

This greatly improved outlook is even better now in the developed countries of the world where most, if not all spinal cord injured patients are taken to designated

*Correspondence: OE Nwankwo, University of Nigeria Teaching Hospital, Enugu, Enugu State, Nigeria spinal centres for management by experts. However, in a developing country like Nigeria, that is not the case because the spinal cord injured, their helpers and relatives are usually in a dilemma as to where to go for treatment, as there are no spinal centres. Invariably they are turned away from ordinary hospitals and asked to go to orthopaedic and teaching hospitals. When they get to these centres, to their consternation and disappointment, they are frequently turned away due to a shortage of hospital beds. ${ }^{4}$ Hilltop Orthopaedic Hospital Enugu, is a privately organised institution manned by orthopaedists. It is in the vicinity of the National Orthopaedic Hospital and the University of Nigeria Teaching Hospital, both in Enugu Metropolis. This hospital accepts spinal cord injured patients for treatment, bringing relief and offering hope to both patients and their relatives. 
It is therefore inundated with these people and is under pressure to receive all new cases. To accommodate as many as possible a 12-week programme of management that includes active participation of the relatives of the patients was developed.

The aims of the programme include:

(1) To give the spinal cord injured the benefit of expert management.

(2) To prevent further damage to the cord and allow for functional recovery especially in those with incomplete cord injury.

(3) To make the patients with residual neurological deficits especially the paraplegics and quadriplegics, as independent as possible by the time of their discharge from the hospital.

(4) To get the relatives informed and closely involved in the management so as to make them understand the possible prognosis and complications and to acquire the basic skills needed to cope with the disabilities of their relation.

The outcome of this programme over a period of 6 years from January 1996 to December 2000 is presented.

\section{Patients and methods}

All the patients with spinal cord injury of less than 2 weeks duration who presented to the hospital and could be admitted within the study period January 1996 to December 2000 are included in the study.

On presentation, clinical and radiological assessment of the injury including degree of neurological involvement is done and patients are categorised into those with incomplete cord injury and those with clinically complete cord injury because of its prognostic importance. The relative who is going to cater for treatment of the patients is then called in for discussion during which the possible prognosis and complications are explained to him.

Finally the condition for admission into the hospital is that this relative agrees to provide a responsible adult aide who will always stay by the patients and help in his treatment and be taught the basic nursing and physiotherapy care of the patients. Also this aide is told the possible prognosis and complications and what his functions will be from the outset.

The programme of treatment proper consists of:

Week 1: (i) full clinical and radiological evaluation of patient to determine whether the cord injury is incomplete or complete, the cord and bony level of injury, presence or not of spinal reflexes particularly the anal reflex, the presence of complications and associated injuries; (ii) resuscitation of the patient; (iii) catheterisation; (iv) institution of the appropriate definitive treatment for the spinal injury eg skull traction for cervical spinal injury and for associated injury; (v) institution of the nursing care and the treatment of complications like pressure sores if already present; (vi) commencement of physiotherapy care; and (vii) neurological re-evaluation is carried out every other day.

Week 2: (i) basic education as regards to the injury and 'morale boosting talks' are started for both the patient and his aide in order to encourage compliance with instructions; (ii) training of the aide in basic nursing care is started.

Week 3: (i) bladder training is started; (ii) training of the aide in basic physiotherapy care eg range of movement exercises for the joints and positioning of limbs to prevent deformities are started.

Week 4: (i) sitting up in bed is started for those with partial injury except for those on skull traction.

Week 5: (i) those with complete injury without any form of neurologic recovery and with the return of reflexes are concluded as paraplegics and quadriplegics. Their relations are informed and are asked to provide wheelchairs; (ii) education and 'morale boosting talks' are intensified to enhance their rehabilitation.

Week 6: (i) in those with cervical injury, skull traction is discontinued and a collar is applied and an $\mathrm{X}$-ray check is done; (ii) sitting up is started for these patients; (iii) ambulation exercise is started for those with partial injury in plaster of Paris lumbar jacket; (iv) bowel regulation training is started for all.

Week 7: (i) those ambulating well are discharged; (ii) wheelchair training is started for paraplegics and quadriplegics.

Week 8: (i) a check X-ray is done for others; (ii) transfer from bed to wheelchair is started; (iii) ambulation is started for those with partial cervical cord injury released from traction.

Week 9: (i) education on how to cope with the home environment is started; (ii) discharge for recovered patients ambulating well.

Week 10: (i) wheelchair training is concluded for most patients; (ii) bladder and bowel movement regulation training is concluded.

Week 11: (i) discharge for rehabilitated quadriplegics; (ii) paraplegics start ambulation exercises on parallel bars with foot-ankle-knee orthosis and proceed to ambulation with crutches.

Week 12: (i) discharge for rehabilitated paraplegics.

\section{Results}

Seventy-four (74) patients met the criteria for admission and were admitted into the study during the 6 years period, January 1996 to December 2000.

Nine patients $(12 \%)$ had incomplete injury of Frankel $\mathrm{C}$ on admission. Out of the nine with incomplete injury, six were quadriparetics while three were paraparetics. Sixty-five $(88 \%)$ had clinically complete injury on admission but 42 were still in the spinal shock phase because they had no spinal reflex activity below the level of the lesion. All were in Frankel group A. Out of the 65, 32 were quadriplegics while 33 were paraplegics. 
A total mortality of eight $(11 \%)$ was recorded. All the deaths occurred in the quadriplegics with complete injury and all died in the first week of admission. These deaths occurred in those with high cervical cord injury at the level of $\mathrm{C} 4$ and above which resulted in respiratory insufficiency.

Eight out of the nine with incomplete cord injury recovered useful neurological function up to Frankel $\mathrm{D}$ and ambulated by the tenth week. One quadriparetic remained in Frankel $\mathrm{C}$ because motor function was hampered by marked hypertonia and hyper-reflexia and he was unable to be ambulated before discharge. By the twelfth week he could be mobilised only by means of a wheelchair. None developed any fixed deformity or pressure sore.

In the group with clinically complete injury, six of those in the spinal shock phase recovered useful neurological function up to Frankel D and were able to ambulate before discharge by the twelfth week. Recovery was observed from the second day to second week in four patients but in two patients recovery was observed from the fourth week. Five out of the six recoveries were initially quadriplegic. Only one was paraplegic with injury at T12 level.

The remaining 51 in the group with clinically complete injury on admission had no neurological recovery. They remained in Frankel A and ended up as quadriplegics and paraplegics.

However, 49 of them were trained to be comfortable with wheelchair use by the time they were discharged by the twelfth week. The remaining two were not conversant with wheelchair use by the twelfth week because of the delay in starting training caused by associated injuries. One had associated severe burns of the chest and abdomen. He was discharged by the sixteenth week while the other had bilateral femoral fracture and had both femora plated and was discharged by the eighteenth week. Only the five young paraplegics with age range 18-24 years were able to ambulate on parallel bars with back-stop. None could ambulate with crutches by the time of discharge by the twelfth week. None of these patients developed fixed deformity. Nine came in with pressure sores including the one with bilateral femoral fracture; these were successfully treated by dressing during their stay.

All patients were weaned to good bowel movement regulation. We had a problem weaning them to good bladder movement regulation without an urethral catheter because of the persistence of residual urine. Most were therefore discharged with an indwelling urethral catheter.

\section{Discussion}

There are accepted conservative and operative methods and guidelines for the treatment of the spinal cord injured with an excellent outcome which have long been established and are well-known to workers in this field of medicine. ${ }^{5-10}$ However, these methods and guidelines and the excellent results achieved are usually done in what one can be equally described as excellent conditions and a near ideal working environment with all the necessary equipment and adequately trained and dedicated staff. It should be borne in mind also that the treatment of the spinal cord injured should be for those with the necessary skill and is teamwork.

This presentation is not to break new grounds but rather to call the attention of fellow workers in this field who are faced with an adverse environment and untrained personnel who are continually challenged by these unfortunate patients that something can be done even in our predicament.

Earlier work in this field in Nigeria by $\mathrm{Eze}^{4}$ gave a lot of inspiration and direction to this study.

Eze, ${ }^{4}$ working in the National Orthopaedic hospital setting, focused on only the rehabilitation of those with complete injury during a 10 -week programme but we decided to extend our focus to both incomplete and complete cord injury and our programme to 12 weeks. The extension of time enabled us to train the aide of the patient and to allow time for the ambulation training of paraplegics.

It was found that informing and involving responsible relatives of the patient early in the management was very helpful. This fostered co-operation and acceptability of the outcome of treatment by them resulting in nonabandonment of their relation. The aide was found to be indispensable in the prevention of bedsores and deformities without which the nursing and physiotherapy staff would not have been able to cope.

It was observed that in order to avoid an unnecessarily long stay of these patients, the programme of their treatment must be timed and enforced with patients and relations being carried along otherwise there is a tendency for them to wait and expect that, with passage of time, recovery back to normal will occur. We employed what we call 'morale boosting talks' to carry the patient and his aide along. These are things purposely said to patients and his aide by the medical staff (doctors, nurses, and physiotherapists, etc) during their interaction to give them encouragement and thereby facilitating their cooperation with the instructions given. This is necessary because the patients and their relations are usually depressed causing loss of zeal and therefore non-compliance with instructions due to ignorance. The talks are not stereotyped but vary both with the medical staff and with the patients and their aides. They also vary with the stage of the illness. The talks were found to be very useful in getting the patients and their aids cooperating fully, especially in nursing and physiotherapy care activities.

The apparently high mortality in the patients with high cervical cord injury with diaphragmatic paralysis is due to our inability to cope with the respiratory insufficiency occasioned by this injury because of lack of respiratory supporting devices.

Six patients recovered neurological function up to Frankel D from the group with clinically complete 
cord injury on admission. These six were among those in spinal shock phase on admission. Obviously they had incomplete cord injury but because they were still in spinal shock, the clinical practice was that of complete cord injury. However, when the shock phase cleared, as signalled by return of spinal reflexes, the true neurological picture of incomplete cord injury manifested. Even though the shock phase was prolonged especially in the two that started having recovery at about the fourth week, this experience has been observed by other workers. One, therefore, should not be hasty in concluding a case as that of a permanent complete cord injury until the spinal reflexes return.

It was not possible to achieve ambulation with crutches in the paraplegics mainly because of the inadequacy of the physiotherapy personnel and constraints of time. Only two part-time physiotherapists were available to us.

However, except for two patients $(3 \%)$ who for obvious reasons of severe associated injuries did not meet our target, the remaining $64(97 \%)$ left the hospital by our target of 12 weeks with varying reasonable level of independence.

Bladder training was started at week 3 to: (i) give room in the programme for the basic education of the patient and his aide regarding his injury and for training the aide in the basic nursing care. This is done in week 2 and, (ii) give enough time for the return of the voiding reflex after spinal shock which is usually about 3 weeks except of course in the lower motor neuron bladder where the voiding reflex never comes back.

Bladder training in this programme consists of the use of an indwelling urethral catheter with continuous drainage method. The urethral meatus and the catheter are cleansed every morning with antiseptic lotion. The patient is catheterised on admission and the catheter and urine bags are changed weekly. By the third week, time-graduated clamping of the catheter is started. We usually start clamping for $15 \mathrm{~min}$ at a time, then gradually progress in $15 \mathrm{~min}$ step-ladder form to $4 \mathrm{~h}$. This usually takes about 2 weeks. The aim is to increase the bladder capacity to hold urine for about $4 \mathrm{~h}$ before reflex voiding is activated.

When this is achieved, training in the method of voiding is started. The type of method now depends on the type of bladder. Patients who have recovered voluntary bladder control in cases of recovering incomplete cord injury are encouraged to unclamp and drain the bladder when the urge to void comes. When this becomes regular, the catheter is removed. In those with upper motor neuron (automatic) bladder, training in reflex voiding is done. The patient is taught to tap the supra-pubic region or stroke their inner thigh to provoke reflex voiding of urine at $4 \mathrm{~h}$ intervals. When this becomes regular after each voiding, residual urine is drained from the bladder and measured and when it is less than $100 \mathrm{ml}$ consecutively for about 2 weeks, the catheter is removed. In the case of lower motor neuron (flaccid) bladder, manual compression of the lower abdomen (Crede's manoeuvre) is employed. The patient is taught how to unclamp the catheter and drain the bladder every $4 \mathrm{~h}$ by gently compressing the lower mid-anterior abdominal wall applying increasing pressure until urine flow stops. After each voiding, residual urine is drained and when it is less than $100 \mathrm{ml}$ consecutively for about 2 weeks, the catheter is removed and the training is completed. All the patients who recovered neurological function recovered normal bladder control before discharge. However, we were unable to achieve regular bladder drainage without catheterisation in the paraplegics and quadriplegics because of the persistence of residual urine after voiding. This, we believe, is achievable if more time is given for the training and practice.

Bowel movement regulation training involves the steps taken to combat constipation and to achieve a 'near normal' pattern of bowel action such that the patient moves the bowel once daily or at least once every 2 days before discharge from the hospital. It is not a rigid method but varies according to individual needs but aims at achieving the same purpose. We usually allow about 4-5 days for the return of spontaneous bowel evacuation. If bowel action returns spontaneously, the patient is encouraged to take high residue diet, stool softening fruits like paw-paw etc, and a lot of fluid to enhance regular motion. In cases where spontaneous bowel evacuation does not return, digital examination of the rectum is done and digital evacuation faeces done at same time. Again the patient is encouraged to take high residue diet, stool softening fruits and a lot of fluid. If the bowel is not moved for 3 consecutive days on this regimen, we give faecal softening drugs such as dioctyl at night to help the patient move the bowel the following day.

This practice is continued to the sixth week. Then by the sixth week, if regular bowel action is not achieved, every other day we introduce use of Bisacodyl suppository given in the night with the aim of producing bowel motion in the morning. This is given for 2 weeks. This usually achieves the desired effect. In the remaining few, we resort to giving a suppository shortly before the time desired for bowel action.

All the aides by the time of discharge from the hospital were conversant with basic nursing care like turning the patient, skin care including use of foam padding at bony points, bowel movement regulation care particularly as regards dietary needs and care of the bladder like antiseptic cleaning of the indwelling catheter and when to change the catheter.

There were also used to giving basic physiotherapy care like joint range of movement exercise and positioning of limbs to prevent deformities and control of wheelchair. With these skills they can help in settling the patient back into the home environment, thus filling the gap of the social worker who is not readily available in our setting. 
We found the outcome of this study encouraging given the circumstances under which it was done and the fact that most of the set objectives were achieved.

\section{Conclusion}

It is concluded that the adoption of this programme would allow the spinal cord injured to get expert treatment within a reasonable time in a less ideal hospital setting where members of the spinal cord injured treatment team are lacking.

Finally, the existence of functional regional spinal centres in a developing country like Nigeria is long overdue. As we are trying to catch up with the world in information technology and other fields, spinal centres should now go beyond the stage of the drawing-board and boardroom politics and come into reality for the benefit of the spinal cord injured.

\section{Acknowledgements}

We are grateful to Dr (Mrs) Joan F Ude, Head of Department of Physiology, College of Medicine, University of Nigeria, Enugu Campus for painstakingly reading through this paper and making necessary grammatical corrections. We are also thankful to $\mathrm{Mr} \mathrm{TO}$ Ubah for his untiring secretarial help.

\section{References}

1 Swain A, Grundy D, Russel J. ABC of the Spinal Cord Injury. Articles published in the BMJ by BMJ Publication, London. 1991; pp 1-3.
2 Ishmael Montane. Historical perspectives of spinal trauma. Spinal Trauma, Philadelphia: J.B. Lippincott, 1991; pp. $1-9$.

3 McSweeney T. Management of Tetraplegia and paraplegia. Postgraduate Textbook of Clinical Orthopaedic. Bristol: Wright, 1993, pp 944-978.

4 Eze CB. A ten-week programme for the hospital management of acute traumatic paraplegia and quadriplegia. Orient Journal of Medicine 1992; 4(4): 93-95.

5 Guttmann L. Surgical aspects of the treatment of traumatic paraplegia. J Bone Joint Surgery (Br) 1949; 31: $399-403$.

6 Bedbrook GM. Spinal injuries with tetraplegia and paraplegia. J. Bone-Joint Surgery (Br) 1979; 61: $267-$ 284.

7 Frankel HL, Hancock DO, Hyslop G. et al. The value of postural reductions in the initial management of closed injuries of the spine with paraplegia and tetraplegia. Paraplegia 1969; 7: 179-192.

8 Holdsworth F. Fractures, dislocations and fracturedislocations of the spine. J Bone Joint Surgery (Am) 1970; 52: $1534-1551$.

9 Bohlam HH. Acute fractures and dislocations of the cervical spine: analysis of thee hundred patients and review of the literature. J Bone Joint Surgery (Am) 1979; 61: $1119-1142$.

10 Bohlam HH. Treatment of fractures and dislocations of the thoracic spine and lumbar spine. J Bone Joint Surgery (Am) 1985; 67: 165-169. 